\title{
ON THE USE OF LEARNING CONTROL FOR IMPROVED PERFORMANCE IN ROBOT CONTROL SYSTEMS
}

\author{
Svante Gunnarsson and Mikael Norrlöf \\ Department of Electrical Engineering \\ Linköping University, S-581 83 Linköping, Sweden \\ www: http://www.control.isy.liu.se \\ email: svante@isy.liu.se, mino@isy.liu.se
}

1997-03-24

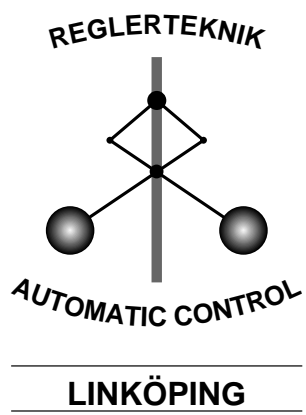

Technical reports from the Automatic Control group in Linköping are available by anonymous ftp at the address 130.236.20.24 (ftp. control.isy.liu.se/pub/Reports/). This report is contained in the compressed postscript file 1934.ps.Z. 


\title{
ON THE USE OF LEARNING CONTROL FOR IMPROVED PERFORMANCE IN ROBOT CONTROL SYSTEMS
}

\author{
S. Gunnarsson, M. Norrlöf \\ Department of Electrical Engineering \\ Linköping University \\ S-58183 Linköping \\ Sweden \\ Fax:+46-13-282622 e-mail: svante@isy.liu.se,mino@isy.liu.se
}

Keywords : Iterative Learning Control, Robotics, Adaptive.

\begin{abstract}
Iterative learning control applied to a simplified model of a robot arm is studied. The iterative learning control input signal is used in combination with conventional feed-back and feed-forward control, and the aim is to let the learning control signal handle the effects of unmodeled dynamics and friction. Convergence and robustness aspects of the choice of filters in the updating scheme of the iterative learning control signal are studied.
\end{abstract}

\section{Introduction}

Iterative learning control (ILC) has been an active research area for more than a decade, mainly inspired by the pioneering work of Arimoto et al, [1]. The fundamental idea in ILC is that the task to be carried out by the system is of repetitive nature, and that the same reference signal is applied several times. Utilizing the repetitive nature of the problem it is possible to adjust the input signal such that the output signal follows the reference signal as closely as possible. Due to the repetitive nature of many operations in robotics this has been a main area of interest in research on and applications of ILC. The topic is discussed from different viewpoints in, for example, [2] and [3].

An important issue in iterative learning control is the convergence properties of the learning procedure, i.e. that the updating procedure of the input signal converges. This issue was addressed already in [1], where some (restrictive) convergence conditions were presented. The convergence properties have then been further studied by several authors. See, for example, [4], [5], [6], [7], [8] and [9].
Even though a main objective for ILC has been to generate an input signal without using prior knowledge of the process to be controlled it turns out that some kind of more or less explicit model of the process is needed in order to design a suitable iterative learning control law. Some approaches to this problem are presented in [10], [11] and [12], where different methods for identifying the system to be controlled are treated.

\section{The Control Problem}

We shall consider the problem of controlling a SISO linear system

$$
Y(s)=G(s) U(s)
$$

where $U(s)$ and $Y(s)$ represent the input and output signals respectively, and $G(s)$ is the transfer function. The system is controlled by combined feed-forward and feedback using

$$
U(s)=F_{f}(s) Y_{D}(s)+F(s)\left(Y_{D}(s)-Y(s)\right)
$$

where $Y_{D}(s)$ denotes the reference signal, and $F_{f}(s)$ and $F(s)$ denote the transfer functions of the feed-forward and the feed-back regulators respectively. We shall consider the control signal generated by the feed-back regulator as error signal, i.e.

$$
E(s)=F(s)\left(Y_{D}(s)-Y(s)\right)
$$

This choice of error signal is motivated by the typical situation in a robot application. Then often $F(s)$ is a PDregulator, which implies that the error signal will be a combination of the position error and the velocity error. Other choices of error signal can of course also be considered. We then get

$$
E(s)=G_{C}(s)\left(\left(G^{-1}(s)-F_{f}(s)\right) Y_{D}(s)\right.
$$


where $G_{C}(s)$ is the transfer function of the closed loop system, i.e.

$$
G_{C}(s)=\frac{F(s) G(s)}{1+F(s) G(s)}
$$

In equation (3) we see that in order to eliminate the error completely we need exact knowledge of the transfer function of the system, that the reference signal can be differentiated sufficiently many times and that $G(s)$ has all its zeros in the left half plane. Without exact knowledge of $G(s)$ it is impossible to achieve that the output follows the desired signal exactly. The error can be reduced by using feed-back, but it can not be eliminated completely.

\section{Iterative Learning Control}

The use of feed-forward and feed-back control will in many cases give very good properties of the robot control system, but in order to further improve the performance and hopefully cope with the problem of unmodeled dynamics we shall consider the use of iterative learning control. It is worth noticing that we here consider ILC as a complement to conventional feed-back and feed-forward control, analogous to for example [2]. In some publications a pure open loop control signal is determined using ILC. We therefore add a correction signal $\Delta U$, see Figure 1 , to the input torque, which thus will be given by

$$
U(s)=F_{f}(s) Y_{D}(s)+F(s)\left(Y_{D}(s)-Y(s)\right)+\Delta U(s)
$$

which gives

$$
E(s)=G_{C}(s)\left(\left(G^{-1}(s)-F_{f}(s)\right) Y_{D}(s)-G_{C}(s) \Delta U(s)\right.
$$

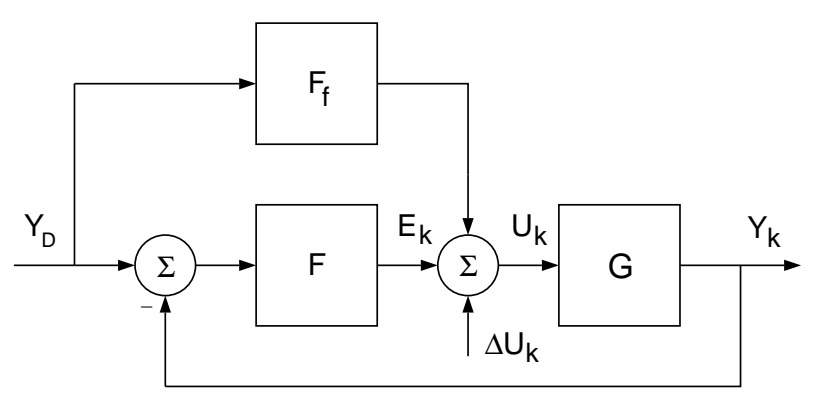

Figure 1: A control system with feed-forward, feed-back and an ILC correction signal

The basic idea in iterative learning control is to use the property that the system carries out the same movement repeatedly, and to iteratively update the correction signal. At iteration $k$ we apply the reference signal $y_{D}(t)$ and the correction signal $\Delta u_{k}(t)$ over some finite time interval. This gives an output signal $y_{k}(t)$ and an error signal $e_{k}(t)$, which then is used to compute a new correction signal for iteration $k+1$.
Considering only linear operations the updating of the correction signal can, in the frequency domain, be expressed as

$$
\Delta U_{k+1}(s)=\sum_{j=0}^{k} \bar{H}_{j}(s) E_{j}(s)
$$

where $\bar{H}_{j}(s), \quad j=0, \ldots, k$ are linear filters. For convenience we shall here however consider recursive update equations on the form

$$
\Delta U_{k+1}(s)=H_{1}(s) \Delta U_{k}(s)+H_{2}(s) E_{k}(s)
$$

The filters $H_{1}(s)$ and $H_{2}(s)$ are used in different ways by different authors. In the original reference [1] the authors use $H_{1}(s) \equiv 1$ and $H_{2}(s)=\gamma \cdot s$, where $\gamma$ is a scalar. The first reference where $H_{1}(s) \neq 1$ is used appears to be [4], where it is shown how $H_{1}(s)$ can be used to obtain less restrictive convergence conditions. In e.g. [13] the case $H_{1}(s)=\mu$, where $\mu<1$ is a scalar, is studied. In [14] ILC of a flexible robot is studied, and some aspects of the choice of the two filters are discussed. A systematic method for filter design using tools from robust control is presented in [15].

\section{Convergence Properties}

We shall now investigate how the error signal behaves when the update equation (9) is applied. Let us first introduce the signal $E_{0}$ defined by

$$
E_{0}(s)=G_{C}(s)\left(\left(G^{-1}(s)-F_{f}(s)\right) Y_{D}(s)\right.
$$

which is the error signal obtained in the first iteration when no correction signal is added, i.e. $\Delta U_{0} \equiv 0$. Using equations (7) and (10) we get

$$
E_{k+1}(s)=E_{0}(s)-G_{C}(s) \Delta U_{k+1}(s)
$$

and inserting equation (9) we obtain

$$
\begin{aligned}
& E_{k+1}(s)=E_{0}(s)-G_{C}(s) H_{1}(s) \Delta U_{k}(s) \\
- & G_{C}(s) H_{2}(s) E_{k}(s) \\
= & \left(1-H_{1}(s)\right) E_{0}(s)+\left(H_{1}(s)-H_{2}(s) G_{C}(s)\right) E_{k}(s)
\end{aligned}
$$

This result can be compared with the error equation in, for example, [14] where the analogous equation for the open loop control case are used. In our case, where the closed loop case is considered, the driving signal in the update equation is $E_{0}(s)$, i.e. the error obtained without the correction signal $\Delta U(s)$.

The convergence properties are determined by the transfer function

$$
H_{E}(s)=\left(H_{1}(s)-H_{2}(s) G_{C}(s)\right)
$$

and referring to [2] the condition for convergence is that

$$
\left|H_{1}(i \omega)-H_{2}(i \omega) G_{C}(i \omega)\right|<1 \quad \forall \omega
$$


Provided that the learning procedure converges the error signal becomes

$$
\bar{E}(s)=\frac{1-H_{1}(s)}{1-H_{1}(s)+G_{C}(s) H_{2}(s)} E_{0}(s)
$$

By plotting the Bode diagram of this transfer function we can illustrate the benefits of applying ILC. We also see that by using $H_{1}(s) \neq 1$ we are not able to eliminate the error completely, but as will be seen later other advantages are obtained by this choice.

\section{A Robot Application}

We shall consider a simplified description of a single robot joint modeled as a double integrator, which means that we have neglected flexibility effects. Initially we also neglect friction, but this will be considered later in the paper. The system is hence given by the differential equation

$$
J \cdot \ddot{y}(t)=u(t)
$$

where $u(t)$ is the input torque, $y(t)$ is the output angle and $J$ is the moment of inertia. Since we shall consider the problem in the frequency domain the system will be represented by the transfer function

$$
G(s)=\frac{1}{J s^{2}}
$$

The feed-forward signal is obtained by multiplying the desired acceleration with the estimated moment of inertia $J^{*}$. In transfer function form this gives

$$
F_{f}(s)=J^{*} s^{2} Y_{D}(s)
$$

The desired acceleration is normally given by a trajectory generator so the computation of the feed-forward signal does not require any differentiation. The feed-back consists of a PD-regulator which means

$$
F(s)=K_{P}+K_{D} s
$$

The error signal $E(s)$ is hence a combination of the position and velocity error, appropriately scaled into a torque signal. Provided that $J^{*}=J$ this control system would give zero error.

\section{SIMULINK Implementation}

The robot model and the control system are implemented in SIMULINK, and the model is simulated using $1 \mathrm{kHz}$ sampling frequency. We shall hence treat the problem using discrete time transfer functions. Since the control signal is constant during the sampling interval the system will by described by the transfer function

$$
G(z)=\frac{T^{2}(z+1)}{2 J(z-1)^{2}}
$$

The discrete time PD-regulator is given by

$$
F(z)=K_{P}+\frac{K_{D}}{T} \frac{(z-1)}{z}
$$

while the feed-forward filter is a double numerical differentiation represented by

$$
F_{f}=\frac{2 J^{*}(z-1)^{2}}{T^{2} z^{2}}
$$

The correction signal will be updated according to

$$
\Delta U_{k+1}(z)=H_{1}(z) \Delta U_{k}(z)+H_{2}(z) E_{k}(z)
$$

where $H_{1}(z)$ and $H_{2}(z)$ are filters that both may be noncausal. See e.g. [14] for an example of the use of noncausal filters.

For evaluation of the ILC methodology in different situations we shall apply the reference trajectory shown in Figure 2. This is a comparatively smooth trajectory and in real robot applications trajectories with sharper acceleration profiles can be expected.

There are several possible ways of evaluating the performance of the control system and we shall here focus on the error (torque) signal defined above. The position and velocity signals, studied separately, are of course also of great interest.

\section{Unmodeled Dynamics}

A main goal is to investigate how the learning control approach can deal with unmodeled dynamics. We shall consider the case when there is an $30 \%$ error in $J^{*}$, i.e. the control system is based on an incorrect value of the moment of inertia.

The properties of the ILC algorithm are determined by the filters $H_{1}(z)$ and $H_{2}(z)$ (and the closed loop system $G_{C}(z)$ ), and the simplest choice is to put both filters equal to unity. It is however well known that these choices will not give a convergent algorithm, and this is also illustrated in Figure 3. The figure shows clearly that the Nyquist curve of $G_{C}(z) H_{2}(z)$, for high frequencies, goes outside the circle with radius and center point equal to one, i.e. the so called learning circle. This is a well known property but this Nyquist curve will later be used for comparison. It is worth noticing that if we apply this choice of filters to the test case described above we initially get a substantial reduction of the error while a continued updating of the correction signal results in an increase of the error. This is an effect of the frequency contents of the reference signal and the character of the Nyquist curve of $G_{C}(z) H_{2}(z)$. Since the Nyquist curve is inside the learning circle for low frequencies there will be an large reduction of the initial error which mainly contains low frequency components. Eventually however the, initially small, high frequency components, corresponding to the part of the Nyquist curve outside the learning circle will have grown so much that they will dominate the error. 
For $H_{1}(z)=1$ the ideal choice of $H_{2}(z)$ would be to choose it as the inverse of $G_{C}(z)$, which, theoretically, would result in convergence to zero in one step. This is however an unrealistic choice since it requires exact knowledge of the closed loop system, i.e. exact knowledge of the system $G(z)$. Since furthermore the gain of $G_{C}(z)$ tends to zero for high frequencies the gain of $H_{2}(z)$ would tend to infinity for high frequencies. The design rule will also result in a non-causal filter, which however not is any limitation. In order to determine a filter $H_{2}(z)$ with more realistic properties we consider

$$
H_{E}(z)=1-H_{2}(z) G_{C}(z)
$$

For all choices of the filter $\mathrm{H}_{2}(z)$ with finite high frequency gain we have to accept that $H_{E}(z)$ tends to one for high frequencies. The ambition will therefore be to ensure that the gain of $H_{E}(z)$ is less than one for all other frequencies. The approach that has been tested is to choose a Butterworth high pass filter $H_{B}(z)$ (here of second order) for which the gain tends to one for high frequencies, and to choose

$$
H_{2}(z)=\hat{G}_{C}^{-1}(z)\left(1-H_{B}(z)\right)
$$

where $\hat{G}_{C}(z)$ denotes the closed loop transfer function we obtain by using the model of the open loop system. Choosing $H_{2}(z)$ according to this design rule, with cutoff frequency of the high pass filter equal to 0.4 times the Nyquist frequency, gives the Nyquist curve depicted in Figure 3. The whole Nyquist curve is now inside the learning circle while it for large frequencies tends to the origin, which is on the border of the learning circle.

The ILC algorithm with $H_{1}(z)=1$ and $H_{2}(z)$ designed as above is then tested in simulations. In Figures 4 and 5 two different methods for evaluating the performance of the algorithm are used. In Figure 4, which shows $\max _{t}\left|e_{k}(t)\right|$ versus iteration number, we see that the error decreases monotonously. Figure 5 shows the spectrum of the error signal $e_{k}(t)$ for different iterations.

Equation (25) gives a filter with relatively high gain for high frequencies. Since the main objective for choosing $H_{2}(z)$ is to obtain a positive phase shift filters with less high frequency gain are of interest. The filter $H_{2}(z)=z$, i.e a pure forward time shift, has also been tested with good results.

\section{Friction}

Since all robots contains some amount of friction it is of interest to evaluate the performance of the ILC algorithm under such conditions. ILC can be seen as a model free method for friction compensation, in contrast to the large number of model based methods that have been discussed in the literature. One example of the use of ILC for friction compensation is presented in [16].

Let us therefore introduce nonlinearities by assuming that the movement of the robot is subject to nonlinear friction. The movements of then robot are then described by

$$
J \ddot{y}(t)=u(t)-f \operatorname{sign}(\dot{y}(t)) \quad \dot{y}(t) \neq 0
$$

and

$$
J \ddot{y}(t)=0 \quad|u(t)| \leq f, \quad \dot{y}(t)=0
$$

where the coefficient $f$ is chosen such that the friction force corresponds to $30 \%$ of the maximum torque. The linear analysis carried out above is not applicable when we have introduced nonlinear elements into the problem, but we can still evaluate the ILC algorithm using simulations. If we carry out the same simulations as in the previous case we get the results shown in Figures 4 and 6 . We still obtain a convergent behavior but the error now converges to a nonzero value.

\section{Extending the Stability Region}

Even though the filter $\mathrm{H}_{2}(z)$ designed above was robust enough to handle that it was designed based on an incorrect value of the moment of inertia it is of interest to further improve the stability margins of the ILC algorithm. This can be done by using both filters $H_{1}(z)$ and $H_{2}(z)$, and we shall here apply the ideas presented in [4]. We therefore introduce a slightly different formulation of the filters and let

$$
H_{1}(z)=\frac{1}{1+V(z)} \quad H_{2}(z)=\frac{1}{1+V(z)} H(z)
$$

where $V(z)$ and $H(z)$ are filters. Inserted in equation (9) this gives the update equation

$$
\Delta U_{k+1}(z)=\frac{1}{1+V(z)}\left(\Delta U_{k}(z)+H(z) E_{k}(z)\right)
$$

In [4] this configuration is used with $H(z)$ constant. The condition for convergence now becomes

$$
\left|1-H\left(e^{i \omega}\right) G_{C}\left(e^{i \omega}\right)\right|<\left|1+V\left(e^{i \omega}\right)\right|
$$

and it is rather obvious that the stability region can be extended by a suitable choice of the filter $V\left(e^{i \omega}\right)$, resulting in a so called stabilizing circle. I e.g. [13] a positive scalar is used, and this choice simply increases the radius of the circle defining the stability region. By letting $V\left(e^{i \omega}\right)$ be frequency dependant the stability region can be extended in a frequency dependant way. It should however be remembered that the prize for the improved stability margins is that the error can not be eliminated completely.

In the simulations we have chosen $V\left(e^{i \omega}\right)$ as a first order high pass filter with cut-off frequency 0.7 times the Nyquist frequency. The high frequency gain of the filter is 0.1 , which means that the stability region is extended in the high frequency regions. The result of this choice is shown in 3 , where the obtained stabilizing circle is shown.

In Figures 4 and 7 the simulation results are shown. The convergence properties are comparable with what was 
obtained without the use of $V\left(e^{i \omega}\right)$ and the error converges to approximately the same level. We have hence achieved the improved robustness without any significant increase in the error level.

\section{Conclusions}

The potential of iterative learning control as a way of improving the performance of robot control systems has been investigated. Convergence and robustness aspects of the choice of design filters have been discussed. The proposed update method of the learning control signal works well also in the presence of nonlinear friction.

\section{Acknowledgments}

This work was supported by CENIIT at Linköping University and by ABB Robotics within ISIS at Linköping University.

\section{References}

[1] S. Arimoto, S. Kawamura, and F. Miyazaki. "Bettering Operation of Robots by Learning". Journal of Robotic Systems, pages 123-140, 1984.

[2] J. Craig. Adaptive Control of Mechanical Manipulators. Addison-Wesley Publishing Company, 1988.

[3] R. Horowitz. "Learning Control of Robot Manipulators". ASME Journal of Dynamic Systems, Measurement, and Control, 115:403-411, 1993.

[4] T. Mita and E. Kato. "Iterative Control and its Application to Motion Control of Robot Arm - A Direct Approach to Servo-Problems". In Proc. 24th CDC, pages 1393-1398, Ft. Lauderdale, Florida, 1985.

[5] F. Padieu and R. Su. "An $H_{\infty}$ approach to Learning Control Systems". International Journal of Adaptive Control and Signal Processing, 4:465-474, 1990.

[6] G. Heinzinger, D. Fenwick, B. Paden, and F. Miyazaki. "Stability of Learning Control with Disturbances and Uncertain Initial Conditions". IEEE Trans. Automatic Control, 37:110-114, 1992.

[7] L. Hideg. Stability of Learning Control Systems. PhD thesis, Oakland University, Rochester, Michigan, 1992.

[8] N. Amann, D.H. Owens, and E. Rogers. "Iterative Learning Control using Optimal Feedback and Feedforward Actions". Technical report, Report Number: 95/13, Centre for Systems and Control Engineering, University of Exeter, Exeter, United Kingdom, 1995.
[9] N. Amann, D.H. Owens, and E. Rogers. "Iterative Learning Control for Discrete Time Systems with Exponential Rate of Convergence". Technical report, Report Number: 95/14, Centre for Systems and Control Engineering, University of Exeter, Exeter, United Kingdom, 1995.

[10] D.M. Gorinevsky. "Direct Learning of Feedforward Control for Manipulator Tracking". In Proc. 1992 IEEE International Symposium on Intelligent Control, pages 42-47, Glasgow, United Kingdom, 1992.

[11] T. Manabe and F. Miyazaki. "Learning Control Based on Local Linearization by Using DFT". Journal of Robotic Systems, 11:129-141, 1994.

[12] D.M. Gorinevsky, D. Torfs, and A.A. Goldenberg. "Learning approximation of feedforward dependence on the task parameters: Experiments in direct-drive manipulator tracking". In Proc. ACC 1995, pages 883-887, Seattle, Washington, 1995.

[13] D. H. Owens. "2D Systems Theory and Iterative Learning Control". In Proc. 2nd European Control Conference, pages 1506-1509, Groningen, Holland, 1993.

[14] S. Panzieri and G. Ulivi. "Disturbance rejection of Iterative Learning Control Applied to Trajectory for a Flexible Manipulator". In Proc. ECC 1995, pages 2374-2379, Rome, Italy, 1995.

[15] D. de Roover. "Synthesis of a Robust Iterative Learning Controller Using an $H_{\infty}$ approach". In Proc. 35th CDC, pages 3044-3049, Kobe, Japan, 1996.

[16] J.S. Liu. "Joint stick-slip friction compensation for robotic manipulators by iterative learning". In Proceedings of the International Conference on Intelligent Robots and Systems, pages 502-509, Munich,Germany, 1994. 


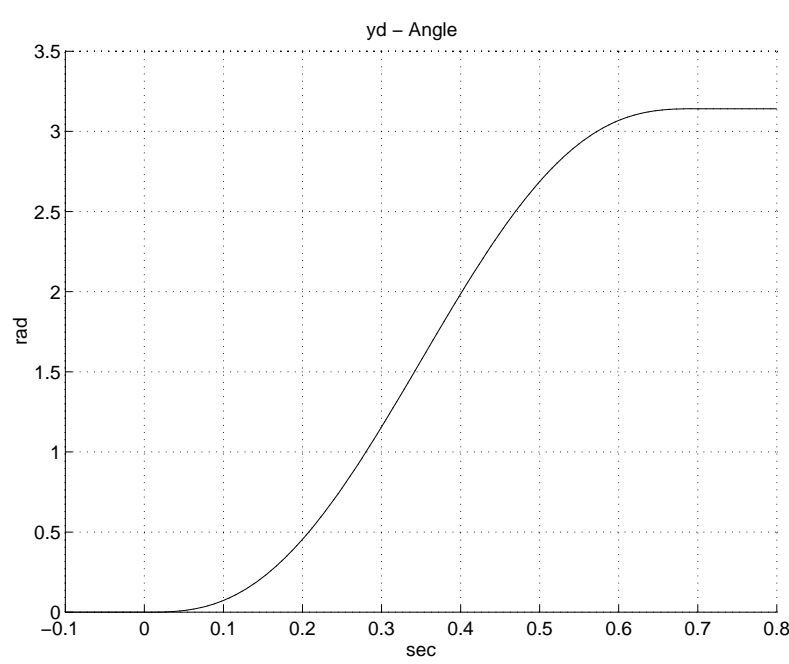

Figure 2: Reference signal

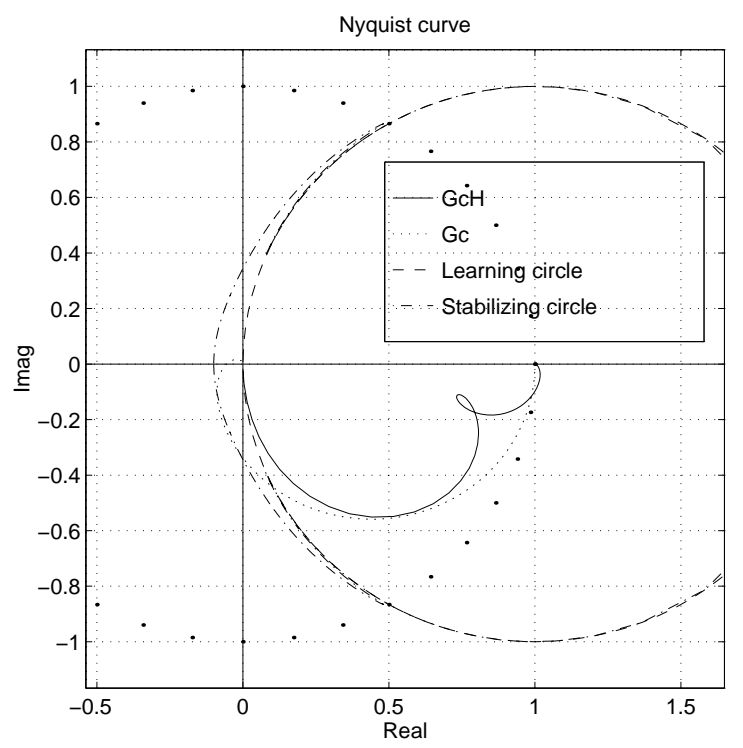

Figure 3: Nyquist curves for $G_{C} H_{2}$ for the choices $H_{2}=1$ and $H_{2}=\hat{G}_{C}^{-1}\left(1-H_{B}\right)$. Learning circle and stabilizing circle.

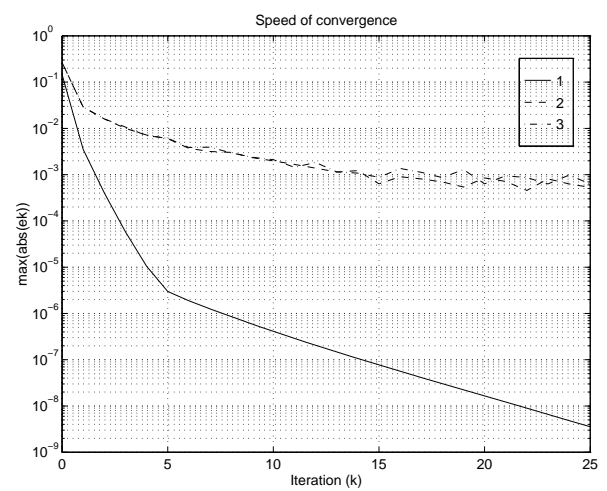

Figure 4: Speed of convergence. 1 - Linear system. 2 System with friction. 3 - System with friction. Learning control with extended stability region.

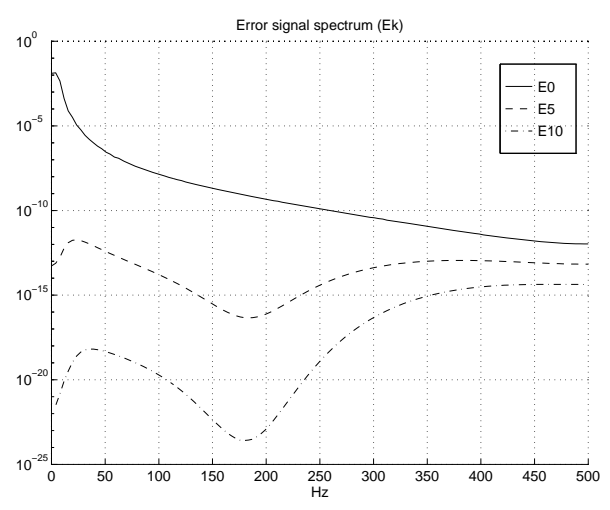

Figure 5: Error signal spectrum for iterations 0,5 and 10. Linear system. $H_{2}=\hat{G}_{C}^{-1}\left(1-H_{B}\right)$.

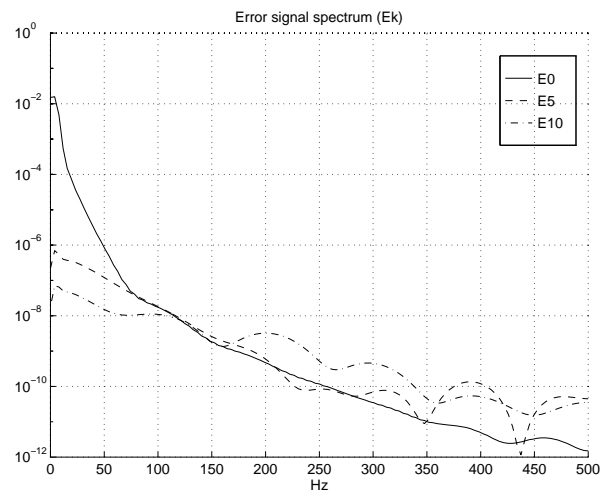

Figure 6: Error signal spectrum for iterations 0,5 and 10. System with friction. $H_{2}=\hat{G}_{C}^{-1}\left(1-H_{B}\right)$.

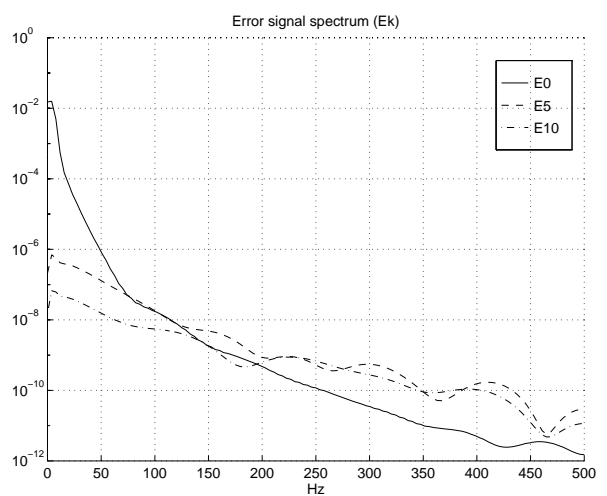

Figure 7: Error signal spectrum for iterations 0,5 and 10. System with friction. $H=\hat{G}_{C}^{-1}\left(1-H_{B}\right) . V$ high pass filter 